\title{
(6) OPEN ACCESS \\ Diurnal variations in luminal and stromal areas of choroid in normal eyes
}

\author{
Takamasa Kinoshita, ${ }^{1,2}$ Yoshinori Mitamura, ${ }^{1}$ Kayo Shinomiya, ${ }^{1}$ Mariko Egawa, ${ }^{1}$ \\ Akiko Iwata, ${ }^{1}$ Akiko Fujihara, ${ }^{1}$ Yoko Ogushi, ${ }^{1}$ Kentaro Semba, ${ }^{1}$ Kei Akaiwa, ${ }^{1}$ \\ Eisuke Uchino, ${ }^{3}$ Shozo Sonoda, ${ }^{3}$ Taiij Sakamoto ${ }^{3}$
}

\begin{abstract}
${ }^{1}$ Department of
Ophthalmology, Institute of Biomedical Sciences,

Tokushima University Graduate School, Tokushima, Japan

${ }^{2}$ Department of Ophthalmology, Sapporo City General Hospital, Sapporo, Japan

${ }^{3}$ Department of

Ophthalmology, Kagoshima University Graduate School of Medical and Dental Sciences, Kagoshima, Japan
\end{abstract}

\section{Correspondence to} Dr Takamasa Kinoshita, Department of Ophthalmology, Institute of Biomedical Sciences, Tokushima University Graduate School, 3-18-15 Kuramoto, Tokushima 7708503, Japan;

knst129@gmail.com

Received 22 February 2016 Revised 2 May 2016 Accepted 22 May 2016 Published Online First 13 June 2016

\begin{abstract}
Aims To determine the diurnal variations of the luminal and stromal areas of the choroid in normal eyes.

Methods This was a prospective observational study of 38 eyes of 38 normal subjects. The blood pressure, heart rate, intraocular pressure and enhanced depth imaging optical coherence tomographic (EDI-OCT) images were recorded every 3 hours between 6:00 and 21:00 hours. The horizontal EDI-OCT images of the subfoveal choroid were converted to binary images. The central choroidal thickness (CCT), total cross-sectional choroidal area, the luminal areas, stromal areas and the ratio of luminal area to total choroidal area (L/C ratio) were determined. Results There were significant diurnal variations in the CCT, total choroidal area, luminal area and $\mathrm{L} / \mathrm{C}$ ratio with the maximum values at $6: 00$ hours and the minimum values at 15:00 hours $(p<0.001$ for the CCT, $p=0.011$ for the total choroidal area, $p<0.001$ for the luminal area and $p=0.014$ for the $L / C$ ratio). There was no significant variation in the stromal area $(p=0.216)$. The range of fluctuation in the CCT was significantly correlated with that in the luminal area and the total choroidal area $(p<0.001)$. However, there was no significant correlation between the fluctuation range in the CCT and that in the stromal area $(p=0.095)$. There was no statistical relationship between the systemic parameters and the choroidal parameters.
\end{abstract}

Conclusions The changes in the luminal area are most likely responsible for the diurnal change in the CCT and subfoveal choroidal area.

Trial registration number UMIN000019060, Pre-results.

\section{INTRODUCTION}

Recent studies have shown that the choroid is closely associated with the physiology of the eye and the pathogenesis of various retinal diseases. ${ }^{1-10}$

Enhanced depth imaging optical coherence tomography (EDI-OCT) and swept-source OCT have enabled clinicians to evaluate the choroidal structure in different kinds of retinal diseases. However, because a layer-by-layer architecture is not distinct, the main structures measured in the OCT images have been the overall choroidal thickness, choroidal area and the shape of the chorioscleral border. CrossMark Only a limited number of studies have assessed the vascular structure of the choroid which unfortunately used custom-made software not in general use. $^{11} 12$

A new method, called the binarisation method, that can differentiate and quantify the choroidal luminal area from the stromal area was recently published. ${ }^{13}$ This method uses an open access software named ImageJ with a detailed protocol. ${ }^{13}$ This method can be used by any researcher with easy accessibility. We used the binarisation technique to differentiate the choroidal luminal area from the stromal area, and we reported that the choroidal thickness and the ratio of luminal area to total choroidal area (L/C ratio) decreased significantly with ageing and longer axial length in normal eyes. ${ }^{14}$

Recent studies have shown that there is a significant diurnal variation in the choroidal thickness. ${ }^{15-17}$ To the best of our knowledge, however, there has not been a study to determine whether changes of the luminal or stromal area of the choroid were mainly responsible for the circadian changes in the choroidal thickness. This information should be useful in understanding the physiology in normal eyes and pathogenesis of chorioretinal diseases.

Thus, the purpose of this study was to determine whether there are diurnal variations in the luminal and stromal areas of the choroid using the binarisation technique.

\section{MATERIALS AND METHODS}

The procedures used conformed to the tenets of the Declaration of Helsinki, and an informed consent was obtained from all of the subjects. This study was approved by the Institutional Review Board of Tokushima University Hospital and registered with the University Hospital Medical Network (UMIN)-clinical trials registry. The registration title is 'UMIN000019060, Choroidal structure on EDI-OCT images’ (17 September 2015).

\section{Inclusion and exclusion criteria}

This was a prospective, cross-sectional, observational study of 50 right eyes of 50 normal, nonsmoking subjects with no ophthalmic or systemic symptoms. The exclusion criteria included the age of $<20$ and $>60$ years, high myopia defined as a refractive error (spherical equivalent) of $<-6.0$ dioptres or an axial length of $>26.5 \mathrm{~mm}$, low EDI-OCT image quality defined as an image quality index of $<30$, abnormal EDI-OCT findings and previous ocular surgeries. Slit-lamp biomicroscopy and indirect ophthalmoscopy were performed to determine whether ocular abnormalities such as corneal diseases, cataracts, vitreoretinal diseases and glaucoma were present. Subjects with any systemic diseases such as hypertension and diabetes mellitus based on the results of their most recent physical examination were also excluded. 


\section{Systemic and ophthalmic examinations}

The systolic blood pressure (SBP), diastolic blood pressure (DBP), heart rate, intraocular pressure (IOP) and EDI-OCT were measured every 3 hours between 6:00 and 21:00 hours. The central corneal thickness, the refractive error and the axial length were measured at 15:00 hours. The SBP, DBP and heart rate were measured on the right arm with a commercial sphygmomanometer (BP-203 RVII, OMRON COLIN, Japan). The mean arterial pressure (MAP) and the mean ocular perfusion pressure (MOPP) were calculated according to the following formulas and used for the analyses: $\mathrm{MAP}=\mathrm{DBP}+1 / 3(\mathrm{SBP}-\mathrm{DBP})$ and $\mathrm{MOPP}=2 / 3 \mathrm{MAP}$ -IOP. All examinations were performed in the sitting position within 10 min for each subject at each time point.

\section{Spectral-domain optical coherence tomography}

Spectral-domain OCT was performed at six-time points with 3-hour intervals; 6:00, 9:00, 12:00, 15:00, 18:00 and 21:00 hours with Spectralis OCT instrument (Heidelberg Engineering, Heidelberg, Germany). Cross-sectional horizontal images of $30^{\circ}$ through the fovea were obtained by EDI-OCT for each eye. Each image was recorded with the eye tracking system without pupil dilation, and 100 scans were averaged to improve the signal-to-noise ratio. The Follow-up mode was used to obtain images of the same position of the fundus for each subject. The first scan which was performed at 6:00 hours was set as the reference for each subject. All subsequent scans were aligned to this. All OCT scans were performed by an experienced orthoptist under standardised mesopic lighting conditions to minimise the possible light-evoked vasodilations and constrictions. On the day of the examination, participants were instructed not to take food, drink and vigorous exercise before the initial examination at 6:00 hours, and then to eat according to their usual daily routine thereafter.

The examined area of the retina and choroid was $1500 \mu \mathrm{m}$ wide and was centred on the fovea. The parameters measured were the central choroidal thickness (CCT), luminal, stromal and total choroidal areas, the central foveal thickness (CFT) and retinal area. The $\mathrm{L} / \mathrm{C}$ ratio was calculated. The $\mathrm{CFT}$ was defined as the distance between internal limiting membrane and the outer surface of the retinal pigment epithelium (RPE), and the $\mathrm{CCT}$ as the distance between the outer border of the RPE and the chorioscleral interface. These distances were measured by two independent investigators (KSh and ME) using the calliper function of the measuring software embedded in the Heidelberg Spectralis OCT. The averages of two measurements were used for statistical analyses.

\section{Evaluation of luminal, stromal and total choroidal areas by binarisation technique}

The EDI-OCT images were evaluated by one of the authors (KSh) who was masked to the clinical findings. The binarisation of the choroidal area in the EDI-OCT images was done by a modified Niblack method using the ImageJ software (ImageJ V.1.47, NIH, Bethesda, Maryland, USA) as described in detail (figure 1). ${ }^{13}$ The examined area was $1500 \mu \mathrm{m}$ wide and located in the subfoveal choroid, and it extended vertically from the RPE to the chorioscleral border. In the binarised images, the light pixels were defined as the stromal areas and the dark pixels were defined as the luminal areas. After including the distance between each pixel, the luminal and stromal areas were automatically calculated. Similarly, the retinal area of $1500 \mu \mathrm{m}$ wide centred on the fovea was determined by measuring the area between internal limiting membrane and the outer border of the RPE.

All parameters were measured three times and the averages were used for the statistical analyses. Although the method for the analysis of the choroid was demonstrated to have a high repeatability and reproducibility in normal eyes, ${ }^{13}$ intra-rater correlation coefficients were calculated for the data obtained from the images recorded at 6:00 hours. The correlations of the OCT parameters with the systemic parameters and IOP were also determined.

\section{Statistical analyses}

Statistical analyses were performed with the SPSS V.22 software (IBM, Armonk, New York, USA). The significances of the diurnal variations in the retinal and choroidal parameters were determined using the repeated-measures analysis of variance

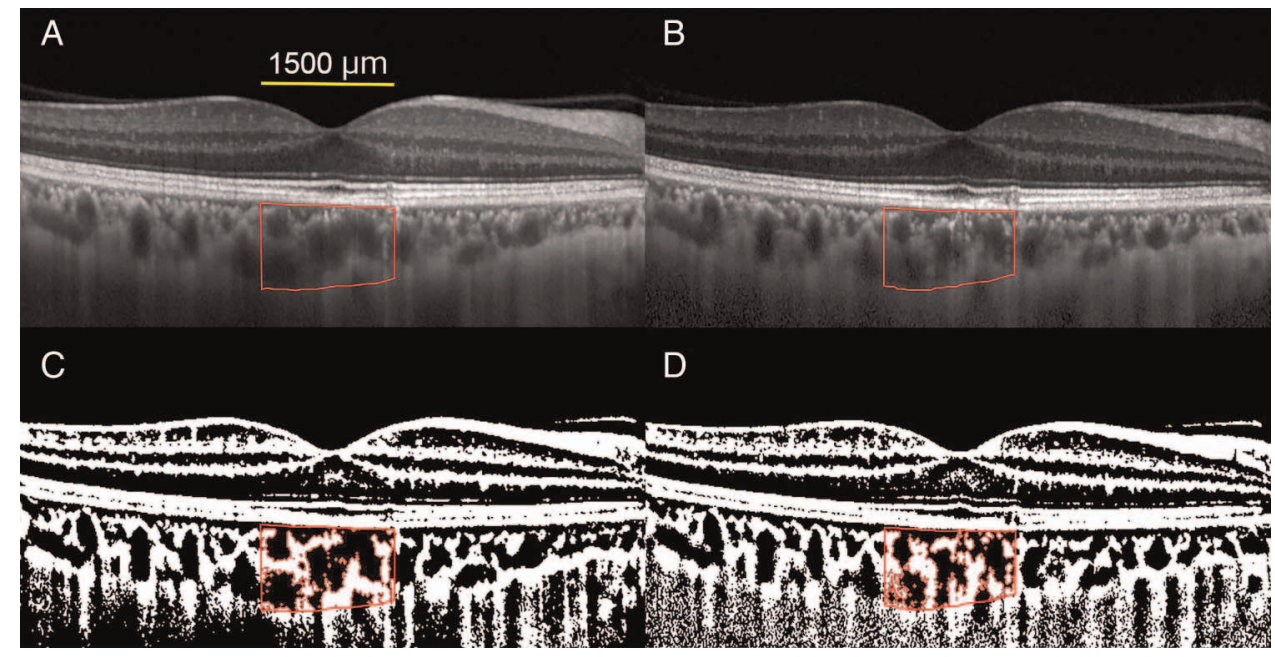

Figure 1 Representative enhanced depth imaging optical coherence tomographic (EDI-OCT) images and converted binary images of a healthy subject. (A and B) Horizontal EDI-OCT images recorded at 6:00 hours (A) and at 15:00 hours (B). The luminal area (dark area) and the stromal area (light area) can be seen. The rectangle surrounded by a red line was excised, and the dark areas were traced by the Niblack method. (C and D) Converted binary images of the EDI-OCT images shown in A (C) and B (D). In the merged binarised images and the margins of traced areas, the light pixels were defined as the stromal area and the dark pixels as the luminal area. The central choroidal thickness (CCT, $325.9 \mu \mathrm{m})$, luminal area (330 146.7 $\mu \mathrm{m}^{2}$ ) and the ratio of luminal to total choroidal area (L/C ratio, 67.8\%) at 6:00 hours were larger than those at 15:00 hours (CCT, $300.7 \mu \mathrm{m}$; luminal area, $261594.3 \mu \mathrm{m}^{2}$; L/C ratio, 59.4\%). 

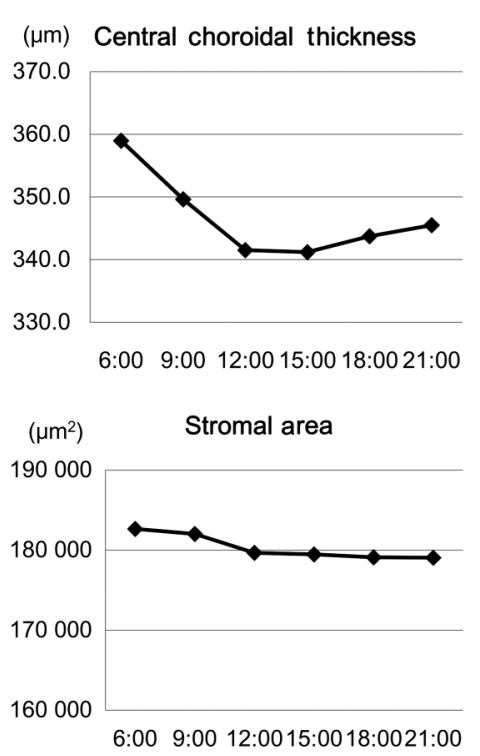
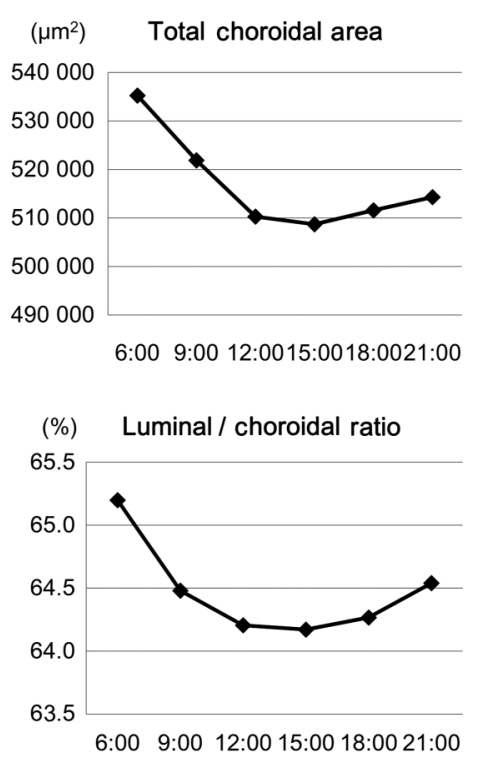
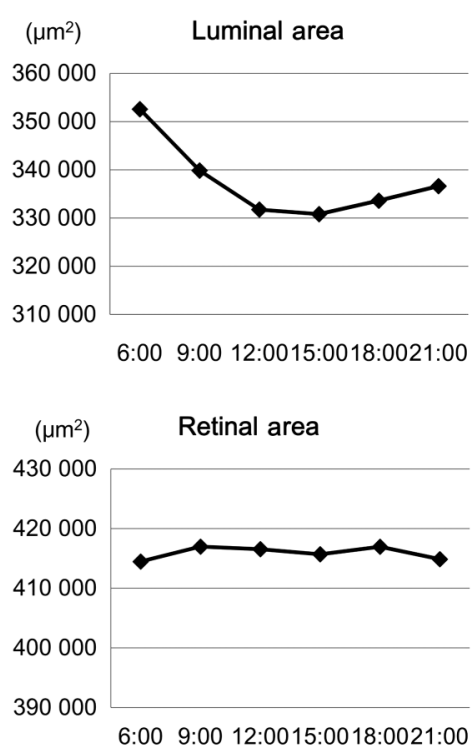

Figure 2 Diurnal variations in the choroidal structural parameters. There were significant diurnal variations in the central choroidal thickness, total choroidal area, luminal area and the ratio of luminal area to total choroidal area. There was no significant diurnal variation in the stromal area and the retinal area.

with Greenhouse-Geisser corrections. The Bonferroni test was used for post hoc analysis. The correlations in the diurnal variation between choroidal OCT parameters and other parameters including the systemic parameters and IOP were determined by calculating the within-subject correlation coefficients using a general linear model. ${ }^{18}$ The intra-rater correlation coefficients were calculated using one-way random effects model for measurements of agreement. The inter-rater correlation coefficients were calculated using two-way mixed effects model for measurements of absolute agreement. A two-sided $\mathrm{p}$ value of $<0.05$ was considered statistically significant.

\section{RESULTS}

\section{Baseline demographic data}

Fifty subjects consisting of 28 men and 22 women were studied. Eleven eyes of 11 subjects were excluded because of high myopia and one eye because of prior laser in situ keratomileusis. No eye was excluded because of low image quality. Thus, the data from 38 eyes of 38 subjects ( 20 men and 18 women) were used for the statistical analyses. The age of the subjects ranged from 21 to 52 years (mean \pm SD, $30.5 \pm 9.11$ years). The axial length, the refractive errors and the central corneal thickness $($ mean $\pm \mathrm{SD})$ were $24.5 \pm 1.07 \mathrm{~mm},-2.8 \pm 2.07$ dioptres and $537.3 \pm 31.18 \mu \mathrm{m}$, respectively.

\section{Repeatability of measurement of OCT parameters}

The intra-rater agreement was high, with an intraclass correlation coefficient of 0.997 (CI 0.994 to 0.998 ) for the total choroidal area, 0.996 (CI 0.993 to 0.998 ) for the luminal area, 0.964 (CI 0.939 to 0.980 ) for the stromal area and 0.974 (CI 0.956 to 0.986 ) for the $\mathrm{L} / \mathrm{C}$ ratio. The inter-rater agreement for the CCT and CFT were 0.997 (CI 0.994 to 0.998 ) and 0.994 (CI 0.989 to 0.997 ), respectively.

\section{Diurnal variations in optical coherence tomographic parameters}

Significant diurnal variations were observed in the mean CCT, the mean total choroidal area, the mean luminal area and the mean $\mathrm{L} / \mathrm{C}$ ratio with the maximum values at 6:00 hours; 359.0 $\pm 76.2 \mu \mathrm{m}$ for the mean CCT, $535243.1 \pm 113295.7 \mu \mathrm{m}^{2}$ for the mean total choroidal area, $352586.1 \pm 113295.7 \mu \mathrm{m}^{2}$ for the mean luminal area and $65.2 \pm 4.13 \%$ of the mean $\mathrm{L} / \mathrm{C}$ ratio (figure 2). The minimum values were found at 15:00 hours; $341.2 \pm 70.1 \mu \mathrm{m}$ for the mean CCT $(\mathrm{p}<0.001), 508696.8$ $\pm 104240.4 \mu \mathrm{m}^{2}$ for the mean total choroidal area $(\mathrm{p}=0.011)$, $330806.8 \pm 85029.5 \mu \mathrm{m}^{2}$ for the mean luminal area $(\mathrm{p}<0.001)$ and $64.2 \pm 3.91 \%$ for the mean $\mathrm{L} / \mathrm{C}$ ratio $(\mathrm{p}=0.014)$.

The mean stromal area was largest at 6:00 hours with a value of $182657.0 \pm 25959.9 \mu \mathrm{m}^{2}$ and smallest at 21:00 hours with a value of $179053.2 \pm 25255.9 \mu^{2}$. However, the difference was not statistically significant $(p=0.216)$. There was no significant variation in the CFT and the retinal area $(\mathrm{p}=0.497$ and $\mathrm{p}=0.441$, respectively).

The difference in the CCT between 6:00 and 15:00 hours was significantly correlated with the differences in the total

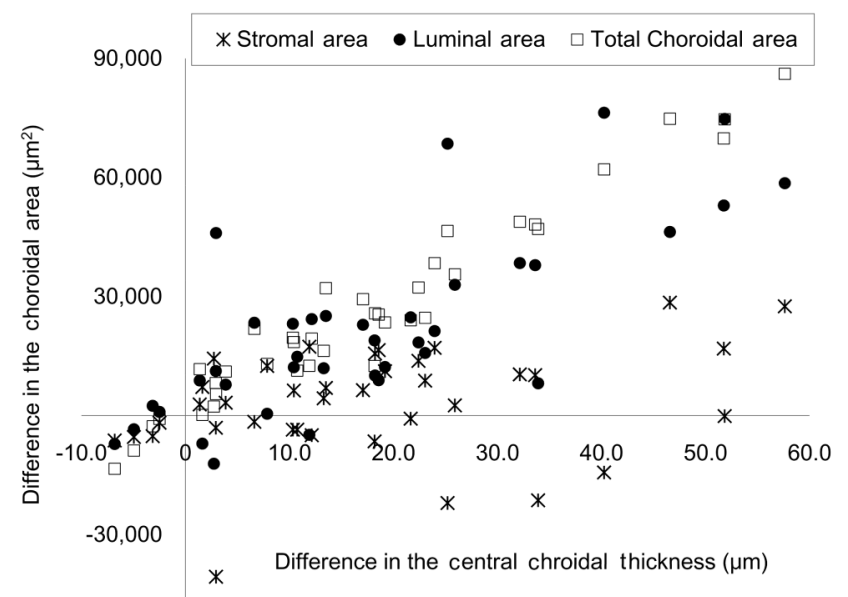

Figure 3 Scatter plots showing the associations between the fluctuation range in the central choroidal thickness and those in the choroidal areas. The difference in the central choroidal thickness between 6:00 and 15:00 hours was correlated with the difference in the total choroidal area and that in the luminal area, but not with that in the stromal area. 


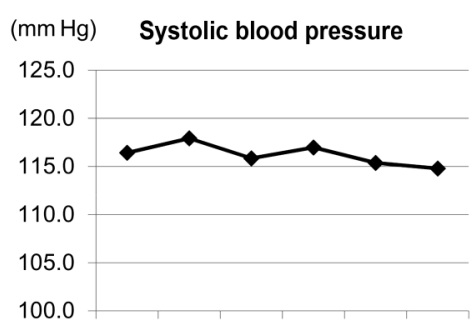

6:00 9:00 12:00 15:00 18:00 21:00

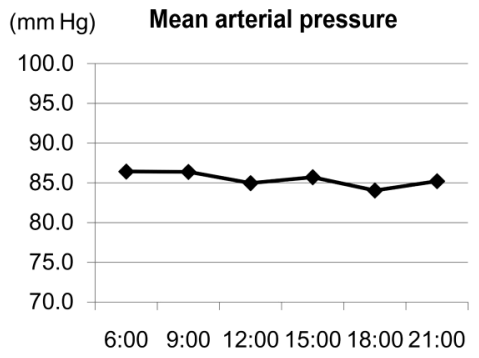

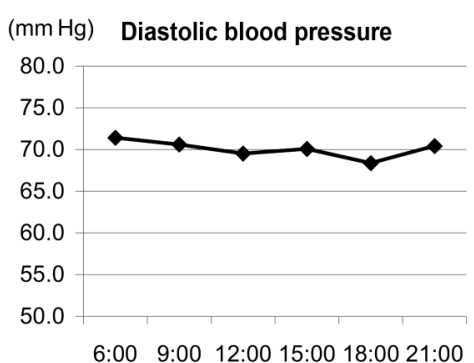

$(\mathrm{mm} \mathrm{Hg})$ Mean ocular perfusion pressure

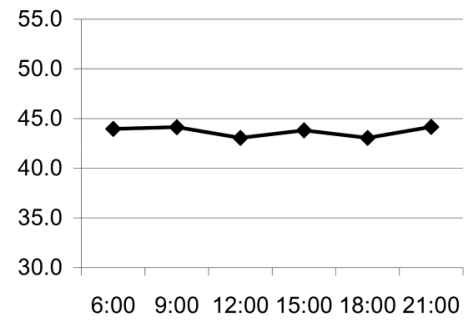

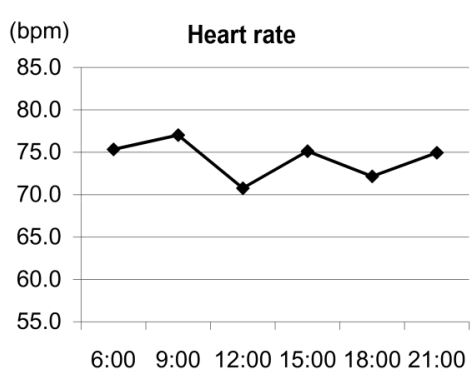

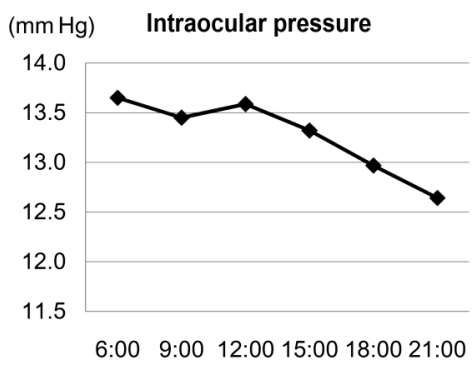

Figure 4 Diurnal variations in the systemic parameters and intraocular pressure. There was no significant diurnal variations in the mean systolic blood pressure, diastolic blood pressure, mean arterial pressure and mean ocular perfusion pressure. There were significant diurnal variations in the mean heart rate and intraocular pressure.

choroidal area $(\mathrm{r}=0.972, \mathrm{p}<0.001)$ and luminal area $(\mathrm{r}=0.767$, $\mathrm{p}<0.001)$ in the partial regression coefficient in which the refractive error and age were set as the control variables (figure 3). However, there was no correlation between the fluctuation range in the CCT and that in the stromal area $(r=0.287, \mathrm{p}=0.095)$.

\section{Diurnal variations in the systemic parameters and intraocular pressure}

None of the subjects had an IOP $>20 \mathrm{~mm} \mathrm{Hg}$ at any time.

There were no significant diurnal variations in the mean SBP, DBP, MAP and MOPP $(p=0.434, p=0.088, p=0.268$ and $p=0.501$, respectively; figure 4$)$. There were significant diurnal variations in the mean heart rate $(\mathrm{p}=0.002)$ and IOP $(\mathrm{p}<0.001)$.

\section{Correlations of OCT parameters with systemic parameters and intraocular pressure}

There were several significant correlations in the diurnal variations between the OCT parameters and the DBP, MAP and IOP but they were not relevant (all $|\mathrm{r}|<0.2$, table 1 ).

\section{DISCUSSION}

Our results showed that there were significant diurnal variations in the CCT, total choroidal area and the luminal area. These parameters were maximal at 6:00 hours, decreased during the day to the minimum values at 15:00 hours and then increased in the evening. These variations are similar to the diurnal variations in the CCT reported in other studies. ${ }^{16} 17$ Our results demonstrated that the choroidal thickness at the fovea and the macular choroidal area had significant diurnal variations.

Importantly, the fluctuations in the stromal area were not significant as well as CFT and retinal area which were used as negative controls. On the other hand, the $\mathrm{L} / \mathrm{C}$ ratio had significant diurnal variations. The change in the CCT was significantly correlated with the change in the luminal area and the total choroidal area. However, there was no correlation between the change in the CCT and that in the stromal area. These results suggest that such fluctuations are mainly due to the diurnal variations in the luminal area. At present, there are reports on the diurnal variations in the choroidal thickness and choroidal
Table 1 Correlations of OCT parameters with systemic parameters and IOP

\begin{tabular}{llllll}
\hline & $\begin{array}{l}\text { Central } \\
\text { choroidal } \\
\text { thickness }\end{array}$ & $\begin{array}{l}\text { Total } \\
\text { choroidal } \\
\text { area }\end{array}$ & $\begin{array}{l}\text { Luminal } \\
\text { area }\end{array}$ & $\begin{array}{l}\text { Stromal } \\
\text { area }\end{array}$ & $\begin{array}{l}\text { L/C } \\
\text { ratio }\end{array}$ \\
\hline SBP & $r=0.111$ & $r=0.075$ & $r=0.110$ & $r=0.082$ & $r=0.019$ \\
& $p=0.127$ & $p=0.300$ & $p=0.131$ & $p=0.260$ & $p=0.795$ \\
DBP & $r=0.139$ & $r=0.128$ & $r=0.154$ & $r=0.025$ & $r=0.088$ \\
& $p=0.055$ & $p=0.077$ & $p=0.033$ & $p=0.733$ & $p=0.226$ \\
HR & $r=0.020$ & $r=0.097$ & $r=0.037$ & $r=-0.004$ & $r=0.024$ \\
& $p=0.788$ & $p=0.181$ & $p=0.612$ & $p=0.957$ & $p=0.744$ \\
IOP & $r=0.152$ & $r=0.088$ & $r=0.160$ & $r=0.050$ & $r=0.094$ \\
& $p=0.036$ & $p=0.224$ & $p=0.027$ & $p=0.488$ & $p=0.197$ \\
MAP & $r=0.139$ & $r=0.116$ & $r=0.148$ & $r=0.055$ & $r=0.064$ \\
& $p=0.055$ & $p=0.110$ & $p=0.041$ & $p=0.452$ & $p=0.382$ \\
MOPP & $r=0.087$ & $r=0.083$ & $r=0.093$ & $r=0.037$ & $r=0.032$ \\
& $p=0.233$ & $p=0.255$ & $p=0.202$ & $p=0.613$ & $p=0.622$ \\
\hline
\end{tabular}

DBP, diastolic blood pressure; HR, heart rate; IOP, intraocular pressure; $L / C$ ratio, ratio of luminal area to total choroidal area; MAP, mean arterial pressure; MOPP, mean ocular perfusion pressure; OCT, optical coherence tomography; SBP, systolic blood pressure.

circulatory parameters, ${ }^{15} 161920$ which make clinicians speculate that the diurnal variation in the choroidal thickness should be related to the diurnal circulatory fluctuations. To the best of our knowledge; however, this is the first report which determined that the diurnal variations in the choroidal thickness and choroidal area should not be attributed to the variation in the stromal area but to the luminal area.

The vascular tone and diameters can be altered by various factors including the blood pressure and myogenic responses, metabolic influences, for example blood gases and $\mathrm{pH}$, visual stimulation and vasoactive agents including angiotensin II and nitric oxide. ${ }^{21}$ Usui et $a l^{16}$ reported a significant negative correlation between the choroidal thickness and SBP during the diurnal variations in these two parameters. Tan et al ${ }^{15}$ reported a significant correlation between the change in the choroidal 
thickness and the change in the SBP. On the other hand, Li et $a l^{22}$ reported that they found no significant correlation between the SBP or DBP and the choroidal thickness. Our results also showed no statistical relationship between the blood pressure and the choroidal parameters. Although the reason for this discrepancy was not determined, the choroidal vessels are under neurogenic control, and the autoregulation of choroidal blood flow is restricted compared with that of the retina. ${ }^{21}$ The degree of fluctuations in the systemic parameters including SBP, DBP, MAP and MOPP were small with no statistical significance. This may explain the discrepancy between the earlier studies and ours. Another possible explanation could be that diurnal variations in the choroid may be influenced by other factors than that examined in this study. Further studies are needed to determine the relationship between choroidal circulatory and structural parameters.

Changes in the IOP could also influence the choroidal parameters. A recent report showed that the diurnal fluctuation of the IOP and choroidal thickness were out of phase, ${ }^{17}$ but no correlation was detected in another report. ${ }^{16}$ In this study, no statistical correlation was found between the choroidal parameters and the IOP. The amplitude of the diurnal fluctuations in the mean IOP was approximately $1.0 \mathrm{~mm} \mathrm{Hg}$. A significant correlation may not have been detected because of the small range of IOP fluctuations.

Evaluating the choroidal structure including the $\mathrm{L} / \mathrm{C}$ ratio should be useful for understanding the pathogenesis of chorioretinal diseases as we reported recently. ${ }^{13} 2324$ However, careful attention should be paid to the diurnal variations in the choroidal structure when evaluating the choroidal structure in various chorioretinal diseases.

There are some limitations in this study. First, we did not examine the 24-hour diurnal variations. The $\mathrm{L} / \mathrm{C}$ ratio during midnight might provide some new information in the future. Second, the scanned area of $1500 \mu \mathrm{m}$ was not enough to assume the diurnal variation of the whole choroid. A wider range of the examination area may be more conclusive.

In conclusion, there are significant diurnal variations in the total choroidal area, luminal area and the $\mathrm{L} / \mathrm{C}$ ratio but not in the stromal area. These findings indicate that the diurnal variations in the choroidal area are mainly due to the fluctuations in the luminal area.

Acknowledgements The authors thank Professor Emeritus Duco Hamasaki of the Bascom Palmer Eye Institute of the University of Miami for providing critical discussions and suggestions for our study and revision of the manuscript.

Contributors All authors have given final approval of this version to be published. Design of the study: TK, YM, ME, SS and TS. Conduct of the study: TK, YM, KSh and ME. Management of the data: YM, KSh, Al, AF, YO, KSe and KA. Analysis of the data: TK, YM, KSh, ME, KSe, EU and SS. Interpretation of the data: TK, YM, $\mathrm{KSh}, \mathrm{ME}, \mathrm{Al}, \mathrm{AF}, \mathrm{YO}, \mathrm{KSe}, \mathrm{KA}$, SS and TS. Preparation of the manuscript: TK, YM, EU, SS and TS. Overall coordination: TS.

Funding This work was supported in part by grant-in-aid 16K11288 (to YM) from the Ministry of Education, Science, Sports and Culture, Japan.

Competing interests None declared.

Patient consent Obtained.

Ethics approval The Institutional Review Board of Tokushima University Hospital.

Provenance and peer review Not commissioned; externally peer reviewed.

Data sharing statement Data are available from the corresponding author upon request.

Open Access This is an Open Access article distributed in accordance with the Creative Commons Attribution Non Commercial (CC BY-NC 4.0) license, which permits others to distribute, remix, adapt, build upon this work non-commercially, and license their derivative works on different terms, provided the original work is properly cited and the use is non-commercial. See: http://creativecommons.org/ licenses/by-nc/4.0/

\section{REFERENCES}

1 Imamura Y, Fujiwara T, Margolis R, et al. Enhanced depth imaging optical coherence tomography of the choroid in central serous chorioretinopathy. Retina 2009;29:1469-73.

2 Maruko I, lida T, Sugano Y, et al. Subfoveal choroidal thickness after treatment of central serous chorioretinopathy. Ophthalmology 2010;117:1792-9.

3 Nakayama M, Keino H, Okada AA, et al. Enhanced depth imaging optical coherence tomography of the choroid in Vogt-Koyanagi-Harada disease. Retina 2012;32:2061-9.

4 Maruko I, lida T, Sugano Y, et al. Subfoveal choroidal thickness after treatment of Vogt-Koyanagi-Harada disease. Retina 2011;31:510-17.

5 Fujiwara T, Imamura Y, Margolis R, et al. Enhanced depth imaging optical coherence tomography of the choroid in highly myopic eyes. Am J Ophthalmol 2009;148:445-50.

6 Ikuno Y, Tano Y. Retinal and choroidal biometry in highly myopic eyes with spectral-domain optical coherence tomography. Invest Ophthalmol Vis Sci 2009;50:3876-80.

7 Chung SE, Kang SW, Lee JH, et al. Choroidal thickness in polypoidal choroidal vasculopathy and exudative age-related macular degeneration. Ophthalmology 2011;118:840-5.

8 Manjunath V, Goren J, Fujimoto JG, et al. Analysis of choroidal thickness in age-related macular degeneration using spectral-domain optical coherence tomography. Am J Ophthalmol 2011;152:663-8.

9 Regatieri CV, Branchini L, Carmody J, et al. Choroidal thickness in patients with diabetic retinopathy analyzed by spectral-domain optical coherence tomography. Retina 2012;32:563-8.

10 Esmaeelpour M, Považay B, Hermann B, et al. Mapping choroidal and retinal thickness variation in type 2 diabetes using three-dimensional 1060-nm optical coherence tomography. Invest Ophthalmol Vis Sci 2011;52: 5311-16.

11 Branchini LA, Adhi M, Regatieri CV, et al. Analysis of choroidal morphologic features and vasculature in healthy eyes using spectral-domain optical coherence tomography. Ophthalmology 2013;120:1901-8.

12 Sohrab M, Wu K, Fawzi AA, et al. A pilot study of morphometric analysis of choroidal vasculature in vivo, using en face optical coherence tomography. PLOS ONE 2012;7:e48631.

13 Sonoda S, Sakamoto T, Yamashita T, et al. Choroidal Structure in Normal Eyes and After Photodynamic Therapy Determined by Binarization of Optical Coherence Tomographic Images. Invest Ophthalmol Vis Sci 2014;55:3893-8.

14 Sonoda S, Sakamoto T, Yamashita T, et al. Luminal and Stromal Areas of Choroid Determined by Binarization Method of Optical Coherence Tomographic Images. Am J Ophthalmol 2015;159:1123-31.

15 Tan CS, Ouyang Y, Ruiz H, et al. Diurnal variation of choroidal thickness in normal, healthy subjects measured by spectral domain optical coherence tomography. Invest Ophthalmol Vis Sci 2012;53:261-6.

16 Usui S, Ikuno Y, Akiba M, et al. Circadian changes in subfoveal choroidal thickness and the relationship with circulatory factors in healthy subjects. Invest Ophthalmol Vis Sci 2012;53:2300-7.

17 Chakraborty R, Read SA, Collins MJ. Diurnal variations in axial length, choroida thickness, intraocular pressure and ocular biometrics. Invest Ophthalmol Vis Sci 2011;52:5121-9.

18 Bland JM, Altman DG. Calculating correlation coefficients with repeated observations: Part 1—correlation within subjects. BMJ 1995;310:446.

19 Iwase T, Yamamoto $K$, Ra E, et al. Diurnal variations in blood flow at optic nerve head and choroid in healthy eyes: diurnal variations in blood flow. Medicine (Baltimore) 2015;94:e519.

20 Pemp B, Georgopoulos M, Vass C, et al. Diurnal fluctuation of ocular blood flow parameters in patients with primary open-angle glaucoma and healthy subjects. $\mathrm{Br} \mathrm{J}$ Ophthalmol 2009;93:486-91.

21 Kur J, Newman EA, Chan-Ling T. Cellular and physiological mechanisms underlying blood flow regulation in the retina and choroid in health and disease. Prog Retin Eye Res 2012;31:377-406.

22 Li XQ, Larsen M, Munch IC. Subfoveal choroidal thickness in relation to sex and axial length in 93 Danish university students. Invest Ophthalmol Vis Sci 2011;52:8438-41.

23 Egawa M, Mitamura Y, Akaiwa K, et al. Changes of choroidal structure after corticosteroid treatment in eyes with Vogt-Koyanagi-Harada disease. $\mathrm{Br} \mathrm{J}$ Ophthalmol 2016;100:1646-50.

24 Egawa M, Mitamura Y, Sano H, et al. Changes of choroidal structure after treatment for primary intraocular lymphoma: retrospective, observational case series. BMC Ophthalmol 2015;15:136. 